\title{
The effect of hexadecaprenol on molecular organisation and transport properties of model membranes ${ }^{\star \otimes}$
}

\author{
Teresa Janas $^{1}$, Krzysztof Nowotarski ${ }^{2}$, Wiesław I. Gruszecki ${ }^{3}$ and Tadeusz Janas ${ }^{2 凶}$ \\ ${ }^{1}$ Biophysics Laboratory, Department of Physics, Technical University, Zielona Góra, Poland; \\ ${ }^{2}$ Department of Biophysics, Institute of Biotechnology and Environmental Sciences, Monte \\ Cassino 21B, 65-561 Zielona Góra, Poland; ${ }^{3}$ Department of Biophysics, Institute of Physics, \\ Maria Curie-Sktodowska University, Lublin, Poland
}

Received: 26 July, 2000; accepted: 03 August, 2000

Key words: long-chain polyprenol, lipid monolayer, bilayer lipid membranes, Langmuir film studies, voltammetric studies.

\begin{abstract}
The Langmuir monolayer technique and voltammetric analysis were used to investigate the properties of model lipid membranes prepared from dioleoylphosphatidylcholine (DOPC), hexadecaprenol $\left(\mathrm{C}_{80}\right)$, and their mixtures. Surface pressuremolecular area isotherms, current-voltage characteristics, and membrane conductance-temperature were measured. Molecular area isobars, specific molecular areas, excess free energy of mixing, collapse pressure and collapse area were determined for lipid monolayers. Membrane conductance, activation energy of ion migration across the membrane, and membrane permeability coefficient for chloride ions were determined for lipid bilayers. Hexadecaprenol decreases the activation energy and increases membrane conductance and membrane permeability coefficient. The results of monolayer and bilayer investigations show that some electrical, transport and packing properties of lipid membranes change under the influence of hexadecaprenol. The results indicate that hexadecaprenol modulates the molecular organisation of the membrane and that the specific molecular area of polyprenol molecules depends on the relative concentration of polyprenols in membranes. We suggest that hexadecaprenol modifies lipid membranes by the formation of fluid microdomains. The results also indicate that electrical transmembrane potential can accelerate the formation of pores in lipid bilayers modified by long chain polyprenols.
\end{abstract}

\footnotetext{
${ }^{\star} 75$ th Anniversary of Membrane Lipid Bilayer Concept.

${ }^{-}$This work was carried out within the research project No. 6 PO4A 01410 supported by the State Committee for Scientific Research in 1996-1998. The financial support of the UNESCO/PAS Polish Network for Cell and Molecular Biology for Krzysztof Nowotarski is gratefully acknowledged.

${ }^{\otimes_{T}}$ Tel.: (48 68) 323 4080; fax: (48 68) 326 5449; e-mail: tjanas@asia.aw.wsp.zgora.pl
}

Abbreviations: DOPC, dioleoylphosphatidylcholine; $\mathrm{C}_{80}$, hexadecaprenol; TLC, thin-layer chromatography; HPLC, high-pressure liquid chromatography. 
Polyisoprenols (polyprenols and dolichols) are found in membrane fractions of prokaryotic and eukaryotic cells (Chojnacki et al., 1987). Polyisoprenols (poly-cis-prenols) are natural products, derivatives of the C5 isoprene unit. The occurrence of $\alpha$-unsaturated polyisoprenols (polyprenols) (Świeżewska et al., 1994; Wanke et al., 1998) and $\alpha$-saturated polyisoprenols (dolichols) (Jankowski et al., 1994; Hemming 1983; Chojnacki et al., 1987) in membrane fractions has been reported. Phosphopolyisoprenols function as hydrophobic carriers of glycosyl residues across membranes in glycosylation reactions (Bugg \& Brandish, 1994). The behaviour of polyisoprenols in model lipid membranes has been studied using several techniques, including ESR (McCloskey \& Troy, 1980; Lai \& Schutzbach, 1984), NMR (de Ropp \& Troy, 1985; Valterson et al., 1985; Knudsen \& Troy 1989), fluorescence spectroscopy (Sunamoto et al., 1983; Boscoboinik et al., 1985; Vigo et al., 1984), X-ray scattering (Gruner, 1985), differential scanning calorimetry (Vigo et al., 1984), voltammetric analysis (Janas et al., 1986 ; 1994; 1998; 2000) and electron microscopy (Janas et al., 2000). However, the mechanism of the interaction between polyprenol and phospholipid molecules is still unclear. Studies of monomolecular films and bilayer lipid membranes containing a mixture of membrane lipids are of considerable importance because of their relevance to numerous natural systems. This paper presents the investigations of monolayer and bilayer lipid membranes modified by a long chain polyprenol - hexadecaprenol $\left(\mathrm{C}_{80}\right)$. The molecule of hexadecaprenol is composed of 16 isoprene units with the structure: $\omega \mathrm{T}_{2} \mathrm{C}_{12} \alpha \mathrm{OH}$, where $\omega$ is an isoprene residue, $\mathrm{T}$ is a trans-isoprene residue, $\mathrm{C}$ is a cis-isoprene residue, $\alpha$ is the $\alpha$-saturated $\mathrm{OH}$ terminal isoprene residue and $\mathrm{OH}$ is the hydroxyl group. Monolayer techniques have been applied to the study of the interactions between dioleoylphosphatidylcholine and other lipids (Gaines, 1966; Costin \& Barnes, 1975; Gruszecki et al., 1999a) and this technique can be used to investigate the origin and magnitude of the molecular interactions in mixed monolayers. The aim of the present work was to study the influence of polyprenol molecules on the organisation and packing of phospholipid molecules in lipid monolayers and bilayers.

\section{MATERIALS AND METHODS}

Chemicals. DOPC (1,2-dioleoyl-sn-glycero3-phosphocholine) was purchased from Sigma. It gave a single spot on Silica Gel thin-layer plates (Merck) in chloroform/methanol/water (65:25:4, by vol.) and in chloroform/methanol/acetic acid/water (50:30: $8: 4$, by vol.). Hexadecaprenol $\left(\mathrm{C}_{80}\right)$ was isolated from leaves of Picea abies (Chojnacki et $a l ., 1975)$ and purified. It gave a single spot on Silica Gel G thin-layer plates (Merck) in ethyl acetate/toluene (5:95, v/v) and on RP-18 HP thin-layer plates (Merck) in acetone. n-Decane and butanol were purchased from Aldrich and Fisher, respectively. The purity of $\mathrm{C}_{80}$ was verified by using a HPLC method (Wanke et al., 1998).

Monolayer formation and isotherm recording. The monolayers were deposited by spreading a proper volume of $\mathrm{C}_{80} / \mathrm{DOPC}$ mixture in chloroform. Surface pressure was measured by the Wilhelmy method (Gruszecki et al., 1999a; 1999b). Monomolecular layers at the air-water interface were formed in a $10 \times$ $40 \mathrm{~cm}$ Teflon trough. The experiments were run at $21^{\circ} \mathrm{C}$. Prior to isotherm recording, monolayers were equilibrated at zero pressure for 5 min to allow evaporation of chloroform. Lipid monolayers were then compressed at a speed of $0.5 \mathrm{~mm} / \mathrm{s}$. Surface pressure was measured by tensiometer PS 3 from Nima Technology and entered into computer memory. Measurement error was less than $0.1 \mathrm{mN} / \mathrm{m}$. Deionised water was used as the subphase. The initial value of the area per molecule was $5 \mathrm{~nm}^{2}$. The obtained data with measurement error less than $0.1 \mathrm{mN} / \mathrm{m}$ were 
further elaborated by the use of Excel 5.0 worksheets (office software package, Microsoft ) and mathematical calculations were performed using Mathematica 3.0 (Wolfram Research).

In the present work we analysed mixed monolayer isotherms in terms of excess free energy of mixing, $\Delta G_{\text {mix }}^{\mathrm{E}}$ was calculated for each mixture using the following equation (Pack et al., 1997; Markowitz et al., 1995):

$$
\Delta G_{m i x}^{E}=\int_{\Pi^{o}}^{\Pi} A_{12} d \Pi-x_{1} \int_{\Pi^{o}}^{\Pi} A_{1} d \Pi-x_{2} \int_{\Pi^{o}}^{\Pi} A_{2} d \Pi
$$

where $A_{12}$ is the area per molecule in the mixed film, $A_{1}$ and $A_{2}$ are molar areas in pure films, $x_{1}$ and $x_{2}$ are molar fractions of component 1 and 2 , and $\Pi$ is the surface pressure. The integrals correspond to the areas under the $\Pi$-A isotherms, and $\Pi^{0}$ is defined as the surface pressure where the monolayer components are ideally miscible. It is generally assumed that $\Pi^{0}$ is close to zero. In practice, $\Pi^{0}$ is commonly set to the lowest measurable surface pressure, and in this work $\Pi^{0}$ was set to $0.4 \mathrm{mN} / \mathrm{m}$. This method involves calculating the differences between the areas under the surface pressure-area isotherms of the mixtures and pure components at a specific surface pressure.

Experimentally it is found that many monolayers can be compressed to pressures considerably higher than their equilibrium spreading pressures. Eventually, however, it is impossible to increase the surface pressure further and the area of the film decreases if constant pressure is maintained or pressure falls in the film held at a constant area. This condition is referred to as the collapse point (collapse pressure $\left(\pi_{\mathrm{c}}\right)$; collapse area $\left(A_{\mathrm{c}}\right)$ ) of the monolayer under the experimental conditions. When collapse occurs, molecules are forced out of the monolayer to form agglomerates of an adjacent bulk phase (Gaines, 1966).

Bilayer formation and electrical measurements. Bilayer lipid membranes in the form of hemispheres were formed according to the technique described previously (Janas et al., 1986) on a Teflon capillary tube in unbuffered ( $\mathrm{pH}$ 6) aqueous solution of $0.1 \mathrm{M}$ and $0.2 \mathrm{M} \mathrm{NaCl}$ (at the inner and outer side of the membrane, respectively). DOPC or $\mathrm{C}_{80}$ /DOPC mixtures used for membrane formation were dissolved in n-decane/butanol $(3: 1, \mathrm{v} / \mathrm{v})$ at $10 \mathrm{mg} / \mathrm{ml}$. The area of the macrovesicular bilayer lipid membrane was about $50 \mathrm{~mm}^{2}$. Saturated silver chloride electrodes were used to apply external voltage and detect the electric potentials. Electrometers were used to measure voltage distribution between the membrane and an external resistance. The area of the membrane, $S$, was determined by optical measurement of membrane dimensions. Temperature, $T$, was controlled by water circulating from an external bath. Electrical conductance of the membrane, $G$, was calculated from current-voltage characteristics.

Membrane permeability coefficients for $\mathrm{Cl}^{-}$ ions, $P_{\mathrm{Cl}^{-}}$, were calculated from the following equation (Tien, 1974):

$$
P_{\mathrm{Cl}^{-}}=\left(E_{\mathrm{Cl}^{-}} \times t_{\mathrm{Cl}^{-}} \times G\right) / F\left(c_{2}-c_{1}\right)
$$

where $c_{1}$ and $c_{2}$ are the concentrations of $\mathrm{NaCl}$ inside and outside the spherical bilayer, respectively; $F$ is the Faraday constant; $E_{\mathrm{Cl}^{-}}$ is the equilibrium potential for $\mathrm{Cl}^{-}$ions; $G$ is specific membrane conductance. The ratio of ionic transference numbers $\left(t_{\mathrm{Na}^{+}} / t_{\mathrm{Cl}^{-}}\right.$for sodium and chloride ions, respectively) was determined from measurements of steady-state diffusion potentials (Janas \& Janas, 1995). Experimental values were fitted by the Goldman-Hodgkin-Katz equation (Gamble et al., 1982; Janas et al., 2000):

$$
\begin{aligned}
& \Delta V_{m}=V_{i}-V_{0}= \\
& (R T / F) \ln \frac{\left(t_{\mathrm{Na}^{+}} / t_{C l}\right) a_{\mathrm{Na}^{+}, \mathrm{o}}+a_{\mathrm{Cl}, i}}{\left(t_{\mathrm{Na}^{+}} / t_{\mathrm{Cl}}\right) a_{\mathrm{Na}^{+}, \mathrm{i}}+a_{\mathrm{Cl}, \mathrm{i}}}
\end{aligned}
$$


which correlates the potential difference, $\Delta V_{\mathrm{m}}$, developed between the two sides of the membrane to the activities, $a_{\mathrm{Na}^{+}}$and $a_{\mathrm{Cl}^{-}}$, of the sodium and chloride ions, respectively, at the inner side ( $i$ ) and the outer side $(o$ ) of the membrane; $V_{\mathrm{i}}$ and $V_{\mathrm{o}}$ are the electric potentials at the inner and outer side of the membrane, respectively; $F$ is the Faraday constant.

The activation energy of ion migration across membrane, $E_{\mathrm{A}}$, was determined from Arrhenius plots of normalized conductance of bilayer lipid membranes (Smith et al., 1984):

$$
\ln \left[(G / C) /\left(G_{0} / C_{0}\right)\right]=\left(E_{\mathrm{A}} / R\right)\left[(1 / T)-\left(1 / T_{0}\right)\right](4)
$$

where: $\ln \left[(G / C) /\left(G_{0} / C_{0}\right)\right]$ is the normalized conductance of the membrane; $G_{0}$ and $C_{0}$ are membrane conductance and membrane capacitance, respectively, at temperature $T_{0}, R$ is the gas constant. The normalisation of membrane conductance (with respect to the membrane capacitance measured simultaneously) corrects for any variations in the bilayer conductance, which are due to variations in bilayer area or bilayer thickness.

\section{RESULTS}

\section{Monolayer experiments}

The Langmuir monolayer technique was used to investigate the interaction of hexadecaprenol with lipid films. Figure 1 shows typical surface pressure-area curves (isotherms) of monolayers prepared from pure hexadecaprenol, pure DOPC, and their mixtures in various molar fractions $(0.01 ; 0.1$; $0.5 ; 0.9 ; 0.99)$ spread on water and compressed at a rate of $0.5 \mathrm{~mm} / \mathrm{s}$ at $21^{\circ} \mathrm{C}$. By changing the molar fraction, a gradual change in the shape of the isotherms is observed. One can notice from Fig. 1 that at pressures below $20 \mathrm{mN} / \mathrm{m}$, monolayers prepared from the molar fraction 0.01 and 0.1 are more expanded than monolayers formed from the molar fractions 0.9 and 0.99 . It means that lipid packing

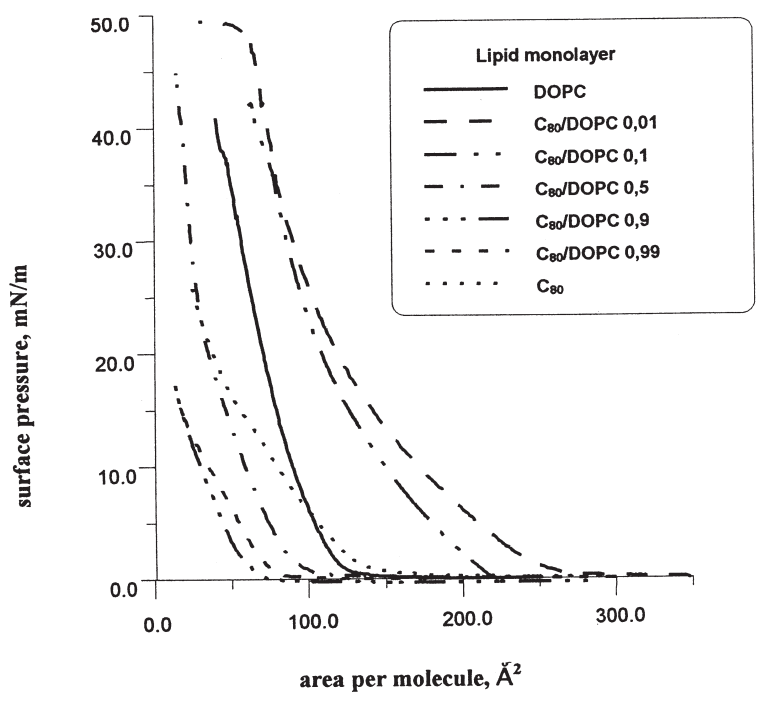

Figure 1. Collection of $\mathrm{C}_{\mathbf{8 0}} / \mathrm{DOPC}$ isotherms for various molar fractions [0-1].

is optimal for membranes of molar fraction equal to 0.9 and 0.99 . Figure 2 shows the dependence of area per molecule, $A^{\pi}$, of the $\mathrm{C}_{80}$ /DOPC monolayer mixture on the molar fraction $\mathrm{C}_{80} / \mathrm{DOPC}$ at constant surface pressures of $2,4,8$ and $16 \mathrm{mN} / \mathrm{m}$. One can see the family of maximal values of area per molecule at $\mathrm{C}_{80}$ /DOPC molar fraction equal to 0.01 .

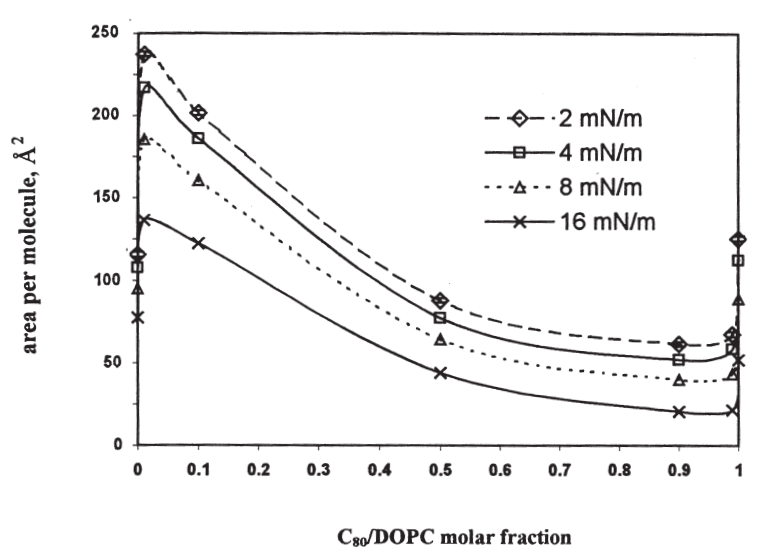

Figure 2. The dependence of area per molecule, $A^{\pi}$, of the $\mathrm{C}_{80}$ /DOPC monolayer mixture on the molar fraction of hexadecaprenol at constant surface pressures of $2,4,8$ and $16 \mathrm{mN} / \mathrm{m}$.

The family of minimal values corresponds to $\mathrm{C}_{80}$ /DOPC molar fraction equal to 0.9 . On the basis of the results presented in Fig. 1, one 
can calculate the specific molecular area, $A_{\mathrm{M}}$, (Costin \& Barnes, 1975; Gruszecki et al., 1999a). The values of $A_{\mathrm{M}}$ are found by extrapolation of the linear parts of the isotherms to zero surface pressure (Fig 3A). The results are shown in Fig. 3B. For pure lipid monolayers the specific molecular area of a hexadecaprenol molecule is estimated to be $99 \pm 5 \AA^{2}$ and the $A_{\mathrm{M}}$ value for a DOPC molecule is 100 $\pm 3 \AA^{2}$. The curve of $A_{\mathrm{M}}$ versus $\mathrm{C}_{80}$ /DOPC molar fraction reaches a minimum for the molar fraction equal to $0.5-0.6\left(43 \pm 3 \AA^{2}\right)$. In addition, we can observe one maximum of $150 \pm 3$ $\AA^{2}$ for the molar fraction equal to 0.01 .

Figure 4 shows the limiting molecular areas, $A_{\infty}$, as a function of $\mathrm{C}_{80} / \mathrm{DOPC}$ molar fraction (Rolland et al., 1996). The $A_{\infty}$ values correspond to the areas occupied by the molecules at the surface pressure equal to $0.4 \mathrm{mN} / \mathrm{m}$. The maximal $A_{\infty}$ value equal to $276 \AA^{2}$ was obtained for the $\mathrm{C}_{80}$ /DOPC molar fraction 0.01 , and the minimal $A_{\infty}$ value equal to $83 \AA^{2}$ for molar fraction 0.99 . From eqn. 1 we have calculated the excess free energy of mixing, $\Delta G_{\text {mix }}^{\mathrm{E}}$, for the surface pressure range 0 to 75 $\mathrm{mN} / \mathrm{m}$. The results are shown in Fig 5 . The excess free energy of mixing has the minimal value equal to $-4.5 \pm 1 \mathrm{~kJ} /$ mole for $\mathrm{C}_{80} / \mathrm{DOPC}$ molar fraction 0.9 and maximal value equal to $8.2 \pm 1 \mathrm{~kJ} /$ mole for $\mathrm{C}_{80} / \mathrm{DOPC}$ molar fraction 0.01. By definition, the excess free energy of mixing for pure lipid monolayers equals 0 $\mathrm{kJ} / \mathrm{mole}$. Figure $6 \mathrm{~A}$ represents the values of collapse pressure, $\pi_{\mathrm{C}}$, for pure $\mathrm{C}_{80}$, pure DOPC and mixed $\mathrm{C}_{80}$ /DOPC monolayers. The collapse pressure of the lipid monolayers of various $\mathrm{C}_{80} / \mathrm{DOPC}$ molar fractions vary from $49.9 \pm 1 \mathrm{mN} / \mathrm{m}$ for pure DOPC monolayer to $26.5 \pm 1 \mathrm{mN} / \mathrm{m}$ for pure $\mathrm{C}_{80}$ monolayer. The maximal value equal to $49.1 \pm 1 \mathrm{mN} / \mathrm{m}$ for $\pi_{\mathrm{C}}$ was obtained for $\mathrm{C}_{80}$ /DOPC molar fraction of 0.01 and the minimal value equal to $15.3 \pm 1$ $\mathrm{mN} / \mathrm{m}$ for $\mathrm{C}_{80} /$ DOPC molar fraction of 0.99 . These results indicate a greater stability of DOPC in comparison with $\mathrm{C}_{80}$ monolayers. Figure $6 \mathrm{~B}$ shows the collapse area $A_{\mathrm{C}}$ as a function of the $\mathrm{C}_{80} / \mathrm{DOPC}$ molar fraction. The collapse area varies from $41.3 \pm 5 \AA^{2}$ for a pure DOPC monolayer to $22.6 \pm 5 \AA^{2}$ for a pure $\mathrm{C}_{80}$ monolayer. The maximal value equal to $63 \pm 5 \AA^{2}$ of $\pi_{\mathrm{C}}$ was obtained for $\mathrm{C}_{80} / \mathrm{DOPC}$
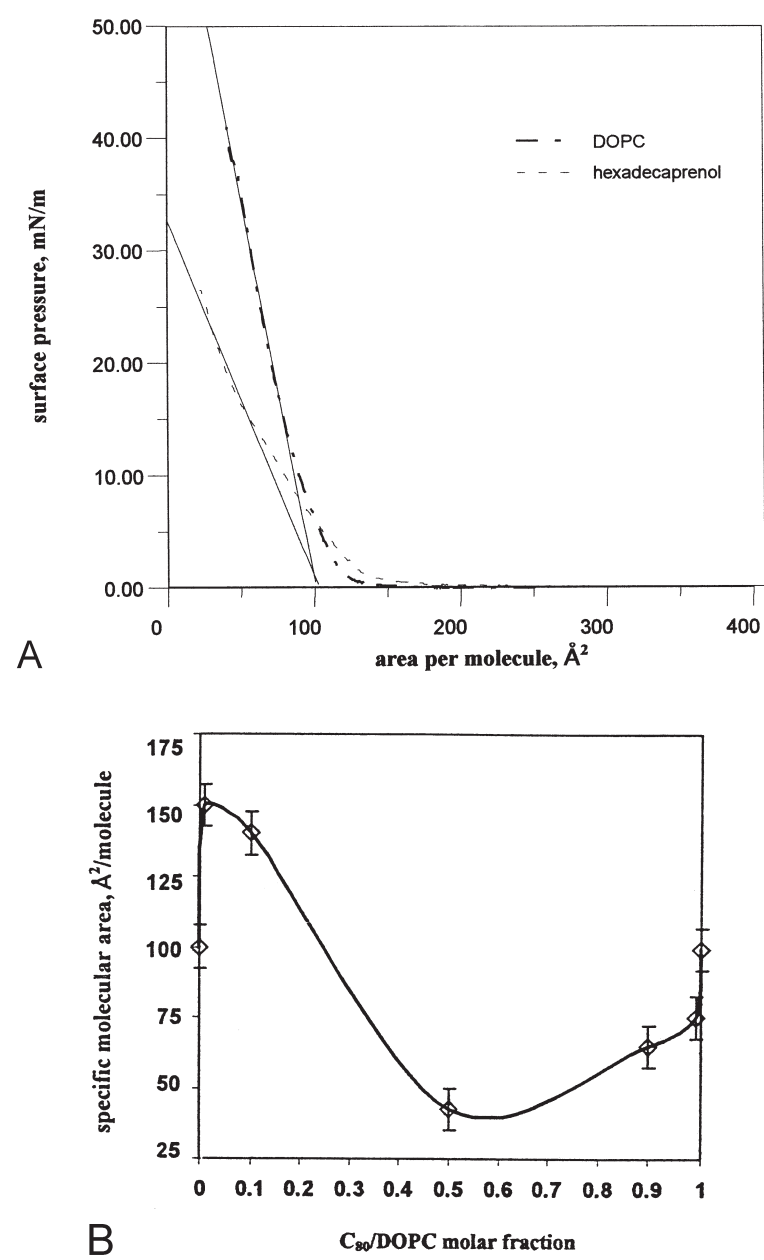

Figure 3A. Isotherms of compression of monomolecular layers of hexadecaprenol and DOPC at the air water interface.

Specific molecular areas $A_{\mathrm{M}}$ are found by extrapolation of the linear parts of the isotherms to zero surface pressure. The $A_{\mathrm{M}}$ values shown are averages of three experiments.

B. Specific molecular area, $A_{\mathrm{M}}$, as a function of molar fractions $\mathrm{C}_{\mathbf{8 0}}$ /DOPC [0-1].

The value of $A_{\mathrm{M}}$ was linearly extrapolated from the linear parts of the isotherms to zero surface pressure.

molar fraction of 0.1 and the minimal one equal to $10.2 \pm 5 \AA^{2}$ for $\mathrm{C}_{80} / \mathrm{DOPC}$ molar fraction of $0.5-0.6$. 


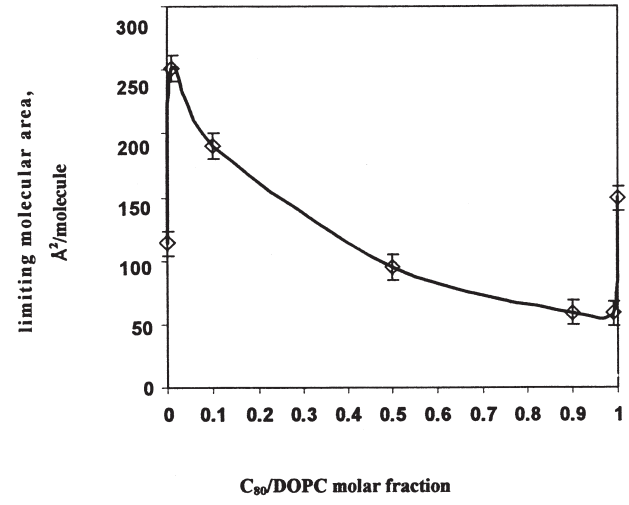

Figure 4. Limiting molecular area, $A_{\infty}$, as a function of molar fraction $\mathrm{C}_{\mathbf{8 0}} / \mathrm{DOPC}$ [0-1].

The values of $A_{\infty}$ were taken for surface pressure of 0.4 $\mathrm{mN} / \mathrm{m}$.

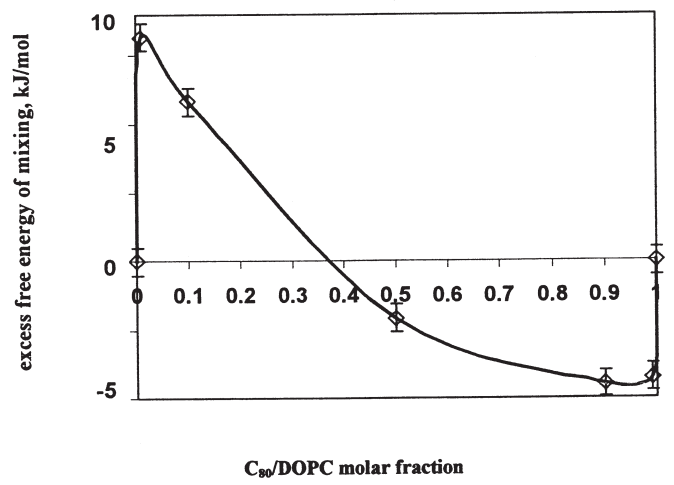

Figure 5. Excess free energy of mixing, $\Delta G_{\text {mix }}^{\mathrm{E}}$, as a function of molar fraction $\mathrm{C}_{80} / \mathrm{DOPC}$ [0-1].

$\Delta G_{\text {mix }}^{\mathrm{E}}$ was calculated for surface pressure of $75 \mathrm{mN} / \mathrm{m}$. The values of $\Delta G_{\text {mix }}^{\mathrm{E}}$ were calculated according to eqn. 1 (see Materials and Methods).

\section{Bilayer experiments}

The behaviour of hexadecaprenol/DOPC membranes as a function of applied potential was studied by performing current-voltage experiments. As presented in Fig. 7A, the curves are symmetric and linear for values in the potential range -20 to $+20 \mathrm{mV}$. The value of the slope increases with the increased percentage of hexadecaprenol in the membrane. The dependence of membrane specific conductance, $G_{\mathrm{S}}$, on the percentage is shown on a semilogarithmic scale in Fig. 7B. The values of $G_{\mathrm{S}}$ increase with the increasing percentage of hexadecaprenol $\left(\mathrm{C}_{80}\right)$ in the membrane. The
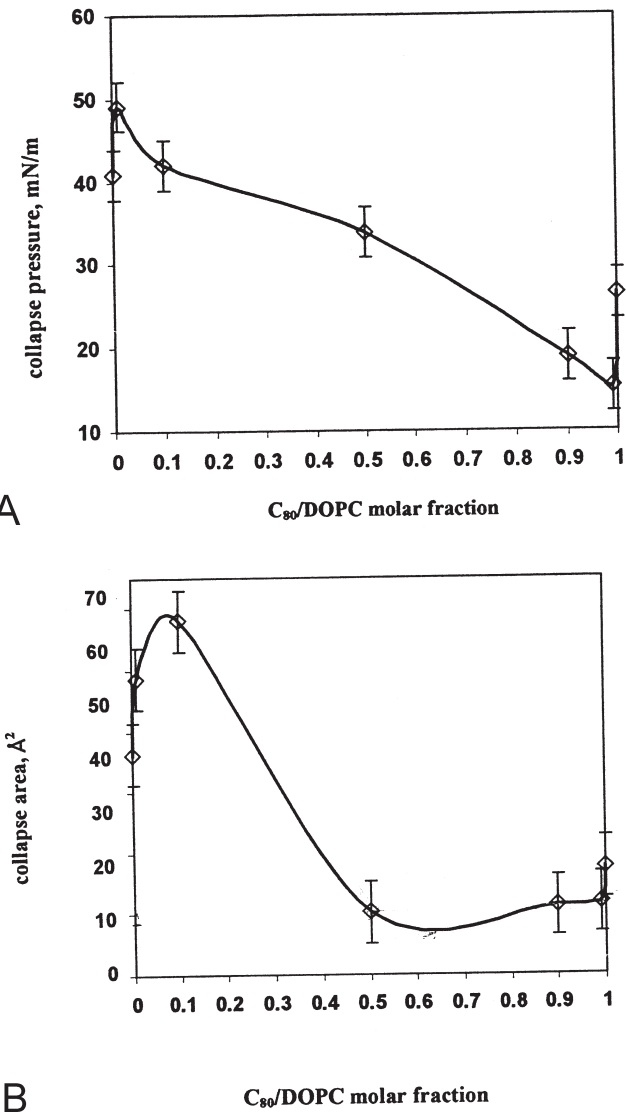

Figure 6. A. Collapse pressure, $\pi_{\mathbf{C}}$, for pure $\mathbf{C}_{\mathbf{8 0}}$, pure DOPC and molar fraction of $\mathrm{C}_{80} / \mathrm{DOPC}$ (0-1). B. Collapse area, $A_{C}$, for mixed lipid monolayers made from $\mathrm{C}_{80} / \mathrm{DOPC}$ at various molar fractions [0-1].

maximal rise, up to $G_{\mathrm{S} \max }=(6.8 \pm 1.1) \times 10^{-7}$ $\mathrm{S} \mathrm{cm}^{-2}$, was observed for the $\mathrm{C}_{80}$ /DOPC mole ratio equal to 0.2 . The value of membrane conductance obtained for DOPC bilayers equals $(4.8 \pm 0.9) \times 10^{-8} \mathrm{~S} \mathrm{~cm}^{-2}$ and is in accordance with the value $(4 \pm 1) \times 10^{-8} \mathrm{~S} \mathrm{~cm}^{-2}$ reported by Gamble et al. (1982). The normalized conductance of bilayer lipid membranes was measured as a function of temperature in the range of $25-42^{\circ} \mathrm{C}$. Typical trends are reported in Fig. 8A. An increase of normalized conductance is observed with increasing temperature. The Arrhenius plots are linear, the slope of the curves depending on the percentage of $\mathrm{C}_{80}$ in the bilayer. The relationship between the value of activation energy of ion transport across the membrane, $E_{\mathrm{A}}$, and the percentage of hexadecaprenol in macrovesicular bilayers is shown in Fig. 8B. The values of activation 

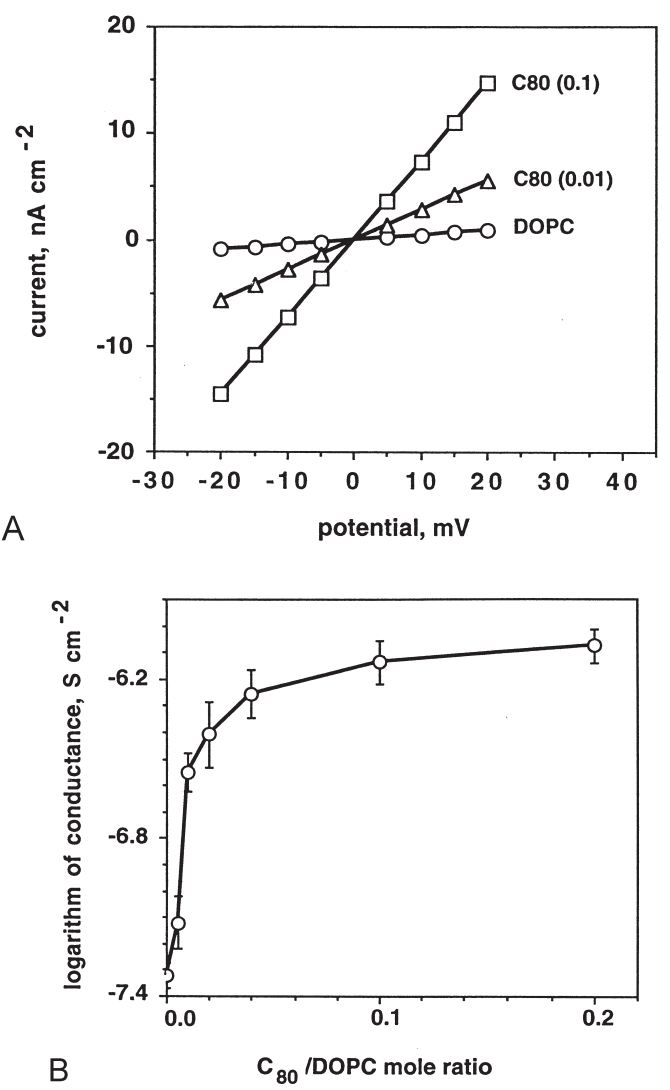

Figure 7A. Current-voltage, I/ $V$, steady-state characteristics of bilayer lipid membranes made from: DOPC (•); $\mathrm{C}_{80} / \mathrm{DOPC}$ mole ratio $0.01(\Delta)$; $\mathrm{C}_{80}$ /DOPC mole ratio $0.1(\bullet)$.

Experiments were performed at $25 \pm 0.1^{\circ} \mathrm{C}$.

B. Specific membrane conductance, $G$, versus $\mathrm{C}_{80}$ /DOPC mole ratio.

Values of membrane conductance were derived from the linear parts of $\mathrm{I} / \mathrm{V}$ curves by least-squares fitting. Each point represents the mean value \pm S.D. obtained for 6-8 different macrovesicular bilayer lipid membranes.

energies were derived from the Arrhenius plots by least squares fitting according to eqn. 4. For lower concentrations of hexadecaprenol, a pronounced decrease of $E_{\mathrm{A}}$ is observed. For higher concentrations of $\mathrm{C}_{80}$ in the membrane, the value of $E_{\mathrm{A}}$ increases slightly. The $E_{\mathrm{A}}$ value decreases from $48 \pm 3$ $\mathrm{kJ} / \mathrm{mole}$ for DOPC bilayers to the value of minimal activation energy, $E_{\mathrm{A} \text { min }}$, equal to 23 $\pm 2 \mathrm{~kJ} / \mathrm{mole}$ for bilayers prepared from mixtures at $\mathrm{C}_{80} / \mathrm{DOPC}$ mole ratio 0.1 and increases slightly for higher concentrations of hexadecaprenol. Smith et al. (1984) reported activation energy equal to $35 \pm 2 \mathrm{~kJ} / \mathrm{mole}$ for a lecithin bilayer. Ionic transference numbers, calculated according to eqn. 3 , were nearly independent of the lipid composition of the membrane and the ratio $\left(t_{\mathrm{Cl}^{-}} / t_{\mathrm{Na}^{+}}\right)$of about 1.5 was obtained at $25^{\circ} \mathrm{C}$. The dependence of the membrane permeability coefficient for chloride ions, $P_{\mathrm{Cl}^{-}}$, on the percentage of
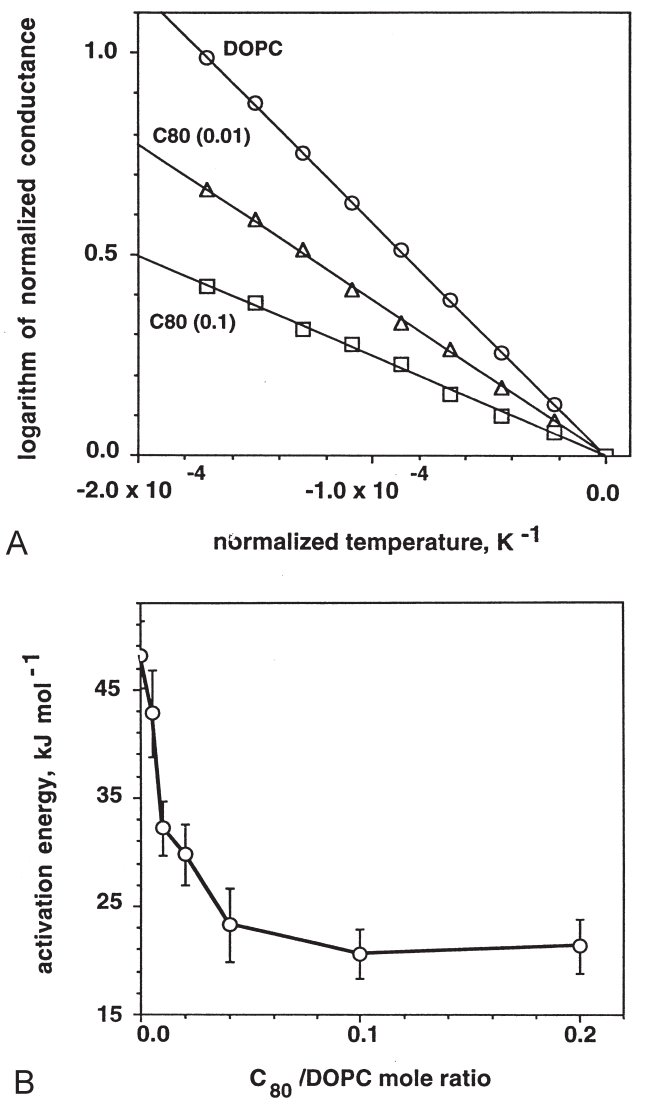

Figure 8A. Arrhenius plots of normalized conductance of macrovesicular bilayer lipid membranes.

Membranes were made from: DOPC (• ); $\mathrm{C}_{80} / \mathrm{DOPC}$ mole ratio $0.01(\Delta) ; \mathrm{C}_{80} / \mathrm{DOPC}$ mole ratio $0.1(\bullet$ ). Logarithm of normalized conductance was calculated as $\log \left[(G / C) /\left(G_{0} / C_{0}\right)\right]$, where $G$ and $C$ represent membrane conductance and membrane capacitance, respectively, at $298 \mathrm{~K}$. Normalized temperature was calculated as: $\left(\mathrm{K}^{-1}\right)-(298 \mathrm{~K})^{-1}$.

B. Activation energy, $E_{\mathrm{A}}$, of ion migration across the membrane vs $\mathrm{C}_{\mathbf{8 0}}$ /DOPC mole ratio.

Values of activation energies were derived from Arrhenius plots by least-squares fitting according to eqn. 4 (see Materials and Methods). Each point represents the mean value \pm S.D. obtained for 6-8 different macrovesicular bilayer lipid membranes. 
hexadecaprenol in the bilayer is shown on a semilogarithmic scale in Fig. 9. The value of $P_{\mathrm{Cl}^{-}}$, calculated according to eqn. 2 , increases with the increasing percentage of $\mathrm{C}_{80}$ in the bilayer. The value of $P_{\mathrm{Cl}^{-}}$equal to $(4.2 \pm$ $0.7) \times 10^{-11} \mathrm{~cm} / \mathrm{s}$ is obtained for a DOPC bilayer. The value of this coefficient for lipid bilayers modified by long chain polyprenols is higher than for DOPC bilayers and the maximal rise, about 30 -fold, is observed for

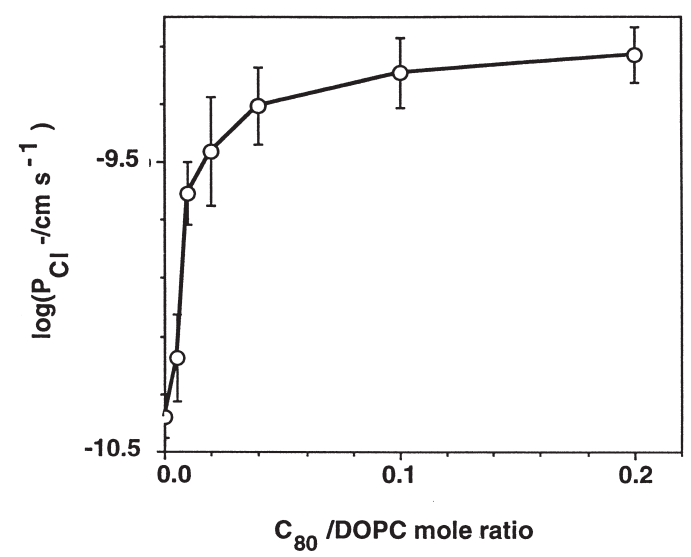

Figure 9. Membrane permeability coefficient for chloride ions, $P_{\mathrm{Cl}^{-}}$, vs $\mathrm{C}_{\mathbf{8 0}}$ /DOPC mole ratio.

Each point represents the mean value \pm S.D. obtained from six to eight different macrovesicular bilayer lipid membranes. Experiments were performed at $25 \pm 1^{\circ} \mathrm{C}$. Values of $\mathrm{P}_{\mathrm{Cl}}$ - were calculated according to the eqn. 2, the values of $t_{\mathrm{Cl}}$ - were calculated according to eqn. 3 (see Materials and Methods).

$\mathrm{C}_{80}$ /DOPC mole ratio equal to 0.2 . For lower concentrations of the polyprenol in the membrane, the rate of increase of $\mathrm{P}_{\mathrm{Cl}}$ - is considerable. For higher concentrations of $\mathrm{C}_{80}$ in the membrane, a slight increase in the value of $\mathrm{P}_{\mathrm{Cl}}{ }^{-}$is observed in comparison with DOPC bilayers.

\section{DISCUSSION}

Lipid membranes modified by hexadecaprenol $\left(\mathrm{C}_{80}\right)$ exhibit different electrical, transport and mixing properties from DOPC monolayers and bilayers. The behaviour of hexadecaprenol-lecithin in monolayers was studied by performing Langmuir monolayer technique experiments. Surface pressurearea isotherms were measured for pure $\mathrm{C}_{80}$, DOPC films and their mixtures. The changes of specific molecular area, $A_{\mathrm{M}}$, free energy of mixing, $\Delta G_{\text {mix }}^{\mathrm{E}}$, and area per molecule at constant surface pressure, $A^{\pi}$, show that hexadecaprenol can modify the structure of lipid aggregates. Similar conclusions were presented for xanthophyll-lecithin monolayers (Tomoaia-Cotişel et al., 1987; N'soukpoé-Kossi et al., 1988). Negelmann et al. (1997) noted that the limiting molecular area value represents the beginning of observable intermodular forces between adjacent molecules in the monolayer. The behaviour of mixed monolayers can be considered in terms of excess free energy of mixing $\Delta G_{\text {mix }}^{\mathrm{E}}$. This interesting phenomenon, represented in Fig. 5, indicates two opposite processes: van der Waals interactions between polyprenol chains, and hydrogen interactions between polyprenol hydroxyl group and the oxygen atom of a water molecule. In the case of a two-component monolayer, the hydrogen bond can be additionally formed between the hydroxyl group of hexadecaprenol and the phosphate group of a DOPC molecule. Our research indicates that for high molar fractions of polyprenols van der Waals forces predominate. This means that the DOPC molecules are monodispersed and surrounded by several $\mathrm{C}_{80}$ molecules each. One can notice that a negative deviation from the rule of additivity is usually interpreted as pointing at a mutual interaction in a two-component system, decreasing the area occupied by molecules in the mixture and lowering the excess of free energy in a monolayer. Positive deviation from the straight line in this case seems to reflect a phenomenon of molecules packed in a monolayer indicating that even small additions of $\mathrm{C}_{80}$ disturb the layer of DOPC. The increase of the area per molecule seems to be the cause of the existence (in the mixture) of the border of phases not occupied by molecules. As can be noticed in the isothermic isobars of Fig. 2, two ex- 
tremes are shown. The curves for surface pressures $2,4,8$ and $16 \mathrm{mN} / \mathrm{m}$ exhibit a maximum of mean area per molecule of the monolayer at molar fraction of $\mathrm{C}_{80}$ equal to 0.01 . This indicates that van der Waals interactions between polyprenyl chains prevail over the attractive interactions between polyprenol and phosphatidylcholine molecules for low molar fractions of hexadecaprenol. It seems that polyprenol molecules form small aggregates in the "sea" of DOPC molecules. We observed that under appropriate conditions the area per molecule of mixed lipid films could increase. The changes depend on the molar fraction of $\mathrm{C}_{80}$ in the membrane indicating that $\mathrm{C}_{80}$ molecules modify the organi- collapse pressure and collapse area represented in Fig. 4A and 4B show that components of the $\mathrm{C}_{80} / \mathrm{DOPC}$ mixed monolayers are miscible.

The family of local minima from Fig. 2 corresponding to the molar fraction of $\mathrm{C}_{80}$ equal to about 0.9 may be understood as illustrating the most packed state of the monolayer and thus aggregation or association of its molecules. This aggregation corresponds to each molecule of DOPC having nine molecules of hexadecaprenol as its neighbours. This could lead to the conclusion that molecules of DOPC during mixing enforce packing of nine $\mathrm{C}_{80}$ molecules around one DOPC molecule and that the process lowers the area per molecule
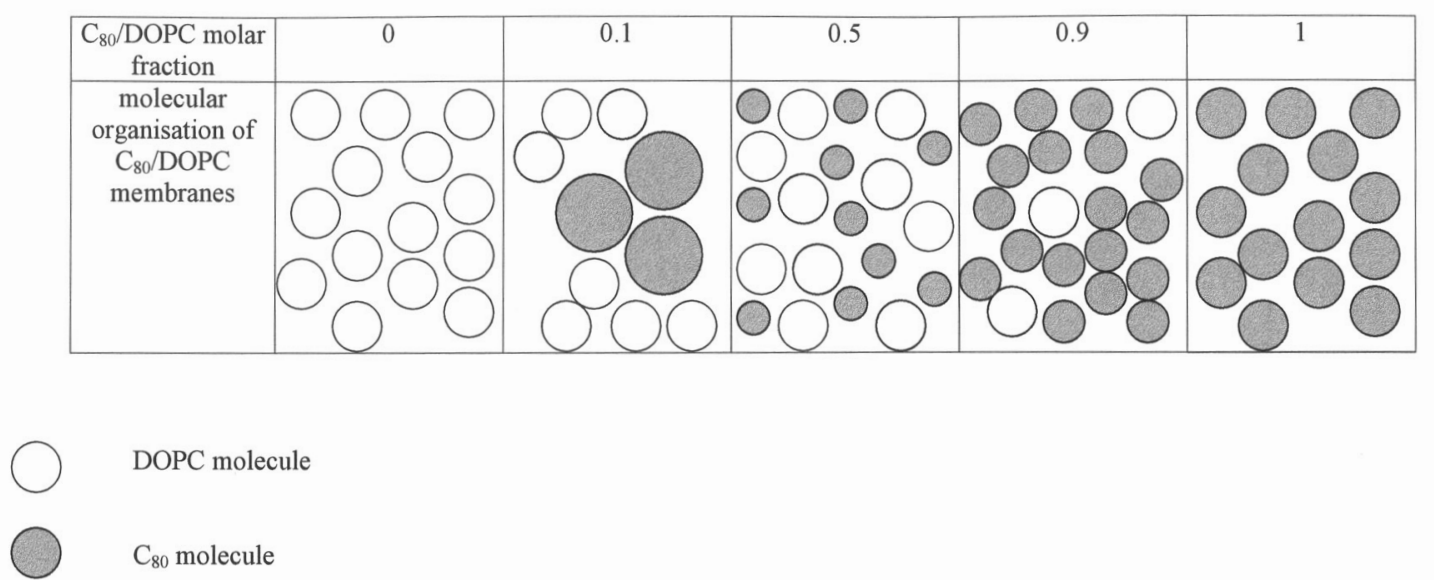

Figure 10. Schematic drawings of a model of the molecular organisation of polyprenol and DOPC in the monolayers investigated.

sation of phospholipid monolayers. The minima in the area per molecule $v s$ molar fraction plots corresponding to the molar fraction of $\mathrm{C}_{80}$ equal to 0.9 may be understood in terms of the appearance of the most packed state of the monolayer caused by the aggregation or association of hexadecaprenol molecules with their neighbours. Another interesting parameter characterising $\mathrm{C}_{80}-\mathrm{DOPC}$ interactions is the miscibility of mixed monolayers (Gaines, 1966). When two components of monolayers are immiscible, mixed monolayers of various compositions will all collapse at the same surface pressure. If components are miscible, collapse pressure will vary with composition. The in the monolayer. Figure 10 presents a hypothetical model of the mixing behaviour of polyprenol and DOPC in the monolayers investigated. The polyprenyl chain is much more flexible than DOPC acyl hydrocarbon chains since it is mainly in cis configuration. Therefore the changes of specific molecular area of mixed $\mathrm{C}_{80}$ /DOPC monolayers can come mainly from changes of specific molecular area of polyprenyl molecules. The changes of $A_{\mathrm{M}}$ for hexadecaprenol show both the modulation of the $A_{\mathrm{M}}$ value of $\mathrm{C}_{80}$ by the surrounding molecules and the tendency to aggregation of $\mathrm{C}_{80}$ molecules at low molar fractions. 
With respect to the electrical properties, the measurements showed that hexadecaprenol increases membrane specific conductance which is consistent with the data for dotriacontaprenol $\left(\mathrm{C}_{160}\right)$ (Janas et al., 2000). Macrovesicular lipid bilayers prepared from hexadecaprenol/DOPC mixtures exhibited much higher permeability for ions in comparison with DOPC bilayers and the values of activation energy of ion transport were much lower than those for DOPC bilayers. The perfect linearity of current-voltage characteristics for hexadecaprenol-containing DOPC bilayers indicates that the increase in membrane permeability and decrease in activation energy are incompatible with the formation of carriers or selective channels by hexadecaprenol molecules. Aggregation of spin-labelled polyisoprenols in phospholipid membranes was observed even at relative concentrations not exceeding 0.005 (McCloskey \& Troy, 1980). These aggregates can modulate the permeability and stability of polyisoprenolphospholipid membranes. Our investigations show that hexadecaprenol substantially decreases the energy barrier for ion migration through membranes, giving rise to an increase of ionic conductance. Lai \& Schutzbach (1984) showed that dolichol promoted membrane leakage in the absence of transmembrane potential in liposomes composed of phosphatidylethanolamine and phosphatidylcholine but not in liposomes composed of phosphatidylcholine only. A strong destabilisation of phosphatidylethanolamine bilayers, but not the phosphatidylcholine ones, in the presence of $\alpha$-saturated polyprenol (dolichol) was detected by Valterson et al. (1985) on studying the phase transition of the bilayers. These experiments were also performed in the absence of transmembrane potential. In contrast to their observation, we report an increase of ion permeability in the presence of transmembrane potential, observed for $\mathrm{C}_{80}$ /DOPC bilayers containing no phosphatidylethanolamine.
The activation energy of ion transport was found to be essentially independent of temperature. This indicates that the influence of temperature on the aggregation behaviour of $\mathrm{C}_{80}$ and DOPC molecules in the membrane is negligible, which is consistent with the observation that the temperature dependence of clustering of polyisoprenol molecules in model membranes is minimal (McCloskey \& Troy, 1980). $\alpha$-Saturated polyprenols were previously found to increase the motional freedom of bilayer lipid membranes (de Ropp \& Troy, 1984; 1985; Vigo et al., 1984; Knudsen \& Troy, 1989) and plasma membranes (Wood et al., 1986; 1989; Schroeder et al., 1987). McCloskey \& Troy (1980) have demonstrated the existence of polyisoprenol clustering in phospholipid bilayers. We suggest the existence in $\mathrm{C}_{80}$ /DOPC bilayers of hexadecaprenol-rich microdomains, which can form transmembrane pores. The microdomain may be stabilized by hydrogen bonds between hydroxyl group of $\mathrm{C}_{80}$ and the ester oxygen of DOPC. These microdomains can modulate the permeability and stability of hexadecaprenol-phospholipid membranes. As analysed in the paper of Smith et al. (1984) the decrease in the activation energy of ion migration across lipid bilayers is related to the increase in the radius of the transmembrane pore. For the value of activation energy of 18 $\mathrm{kJ} /$ mole, the authors estimated the minimum pore radius to be about $1 \mathrm{~nm}$. For $\mathrm{C}_{80} / \mathrm{DOPC}$ bilayers, with the activation energy for ion transport about 2-fold lower, the minimal pore radius can be estimated to be around $2 \mathrm{~nm}$. The action of $\mathrm{C}_{80}$ seems therefore to affect the formation of these pores and to increase their size. The function of polyprenyl microdomains in biological membranes may also depend on their ability to induce local changes in membrane thickness and membrane fluidity corresponding to the hydrophobic thickness and environment requirements of an integral membrane protein located in such domain, in accordance with the mattress model (Mouri- 
tsen \& Bloom, 1984) of lipid-protein interactions in membranes.

In conclusion, the results of monolayer and bilayer investigations show that some electrical, transport and packing properties of lipid membranes change under the influence of hexadecaprenol. The results indicate that: hexadecaprenol modulates the molecular organisation of the membrane; the specific molecular area of polyprenol molecules depends on the relative concentration of polyprenols in membranes; hexadecaprenol can modify lipid membranes by the formation of fluid microdomains. The results also indicate that electrical transmembrane potential can accelerate the formation of pores in lipid bilayers modified by long chain polyprenols.

The authors would like to thank Prof. Tadeusz Chojnacki and Dr. Ewa Świeżewska from the Department of Lipid Biochemistry, Institute of Biochemistry and Biophysics, Polish Academy of Sciences, Warsaw, for stimulating discussions.

\section{R E F E R E N C E S}

Boscoboinik, D.O., Feliz, M., Disalvo, A. \& Belocopitow, E. (1985) The effect of dolichol on the permeability properties of phosphatidylcholine bilayers. Chem. Phys. Lipids 38, 343-352.

Bugg, T.D.H. \& Brandish, P.E. (1994) From peptidoglycan to glycoproteins: Common features of lipid-linked oligosacharide biosynthesis. FEMS Microbiol. Lett. 119, 205.

Chojnacki, T., Jankowski, W., Mankowski, T. \& Sasak, W. (1975) Preparative separation of naturally occuring mixtures of polyprenols on hydroxyalkoxypropyl-sephadex. Anal. Biochem. 69, 114-119.

Chojnacki, T., Świeżewska, E. \& Vogtmann, T. (1987) Polyprenols from plants - structural analogues of mammalian dolichols. Chem. Scripta 27, 209.
Costin, I.S. \& Barnes, G.T. (1975) Two-component monolayers. J. Colloid Interface Sci. $\mathbf{5 1}$ 106-121.

de Ropp, J.S. \& Troy, F.A. (1984) Chemical synthesis and ${ }^{2} \mathrm{H}$ NMR investigation of polyisoprenols: Dynamics in model membranes. Biochemistry 23, 2691-2695.

de Ropp, J.S. \& Troy, F.A. (1985) ${ }^{2}$ H NMR Investigation of the organisation and dynamics of polyisoprenols in membranes. J. Biol. Chem. 260, 15669-15674.

Gaines, G.L. (1966) Insoluble Monolayers at Liquid-Gas Interfaces; pp. 281-301, John Wiley, New York.

Gamble, F., Robello, M., Usai, C. \& Marchetti, C. (1982) Properties of ionic transport through phospholipid-glycolipid artificial bilayers. Biochim. Biophys. Acta 693, 165-172.

Gruner, S.M. (1985) Intristic curvature hypothesis for biomembrane lipid composition: A role for nonbilayer lipids. Proc. Natl. Acad. Sci. U.S.A. 82 3665-3669.

Gruszecki, W.I., Sujak, A., Strzałka, K., Radunz, A. \& Schmid, G.H. (1999a) Organisation of xanthophyll-lipid membranes studied by means of specific pigment antisera, spectrophotometry and monomolecular layer technique. Lutein versus zeaxanthin. Z. Naturforsch. 54c, 517-525.

Gruszecki, W.I., Grudziński, W., Banaszek-Glos, A., Matula, M., Kernen, P., Krupa, Z. \& Sielewiesiuk, J. (1999b) Xantophyll pigments in light-harvesting complex II in monomolecular layers: Localisation, energy transfer and orientation. Biochim. Biophys. Acta 1412, 173-183.

Hemming, F.W. (1983) Biosynthesis of dolichol and related compounds; in Biosynthesis of Isoprenoid Compounds (Porter, J.W. \& Spurgeon, S.L., eds.) p. 305, John Wiley, New York.

Janas, T. \& Janas, T. (1995) Interaction of undecaprenyl phosphate with phospholipid bilayers. Chem. Phys. Lipids 77, 89-97.

Janas, T., Kuczera, J., Chojnacki, T. \& KrajewskaRychlik, I. (1986) Properties of lecithin-dodecaprenol macrovesicular bilayer membranes. Chem. Phys. Lipids 39, 347-355. 
Janas, T., Kuczera, J. \& Chojnacki, T. (1989) Voltammetric analysis of polyisoprenoid-containing bilayer lipid membranes. Chem. Phys. Lipids 51, 227-238.

Janas, T., Chojnacki, T., Świeżewska, E. \& Janas, T. (1994) The effect of undecaprenol on bilayer lipid membranes. Acta Biochim. Polon. 41, 351-357.

Janas, T., Walińska, K. \& Janas, T. (1998) Electroporation of polyprenol-phosphatidylcholine bilayer lipid membranes. Bioelectrochem. Bioenerg. 45, 215-220.

Janas, T., Walińska, K., Chojnacki, T., Świeżewska, E. \& Janas, T. (2000) Modulation of properties of phospholipid membranes by the longchain polyprenol $\left(\mathrm{C}_{160}\right)$. Chem. Phys. Lipids 106, 31-40.

Jankowski, W.J., Świeżewska, E., Sasak, W. \& Chojnacki, T. (1994) Occurence of polyprenols and dolichols in plants. J. Plant Physiol. 143, 448-452.

Knudsen, M.J. \& Troy, F.A. (1989) Nuclear magnetic resonance studies of polyisoprenols in model membranes. Chem. Phys. Lipids 51, 205-212.

Lai, C.S. \& Schutzbach, J.S. (1984) Dolichol induces membrane leakage of liposomes composed of phosphatidylethanolamine and phosphatidylcholine. FEBS Lett. 169, 279282.

Markowitz, M.A., Puranik, D.B. \& Singh, A. (1995) Monolayer study of diacetylenic phosphatidylcholine and phospholipids containing metal-chelating iminodiacetic acid headgroup. Chem. Phys. Lipids 76, 63-71.

Mouritsen, O.G. \& Bloom, M. (1984) Mattress model of lipid-protein interactions in membranes. Biophys. J. 46,141-153.

McCloskey, M.A. \& Troy, F.A. (1980) Paramagnetic isoprenoid carrier lipids. Biochemistry 19, 2056-2060.

N'soukpoé-Kossi, C., Sielewiesiuk, J., Leblanc, R.M., Bone, R.A. \& Landrum, J.T. (1988) Linear dichroism and orientational studies of carotenoid Langmuir-Blodgett films. Biochim. Biophys. Acta 940, 255-265.
Negelmann, L., Pisch, S., Bornscheuer, U. \& Schmidt, R. (1997) Properties of unusual phospholipids. III: Synthesis, monolayer investigations and DSC studies of hydroxy octadeca(e)noic acids and diacylglycerophosphocholines derived therefrom. Chem. Phys. Lipids 90, 117-134.

Pack, D.W. \& Arnold, F.H. (1997) Langmuir monolayer characterization of metal chelating lipids for protein targeting to membranes. Chem. Phys. Lipids 86, 135-152.

Rolland, J.P., Santaella, C. \& Vierling, P. (1996) Molecular packing of highly fluorinated phosphatidylcholines in monolayers. Chem. Phys. Lipids 79, 71-77.

Schroeder, F., Gorka, C., Williamson, L.S. \& Wood, W.G. (1987) The influence of dolichols on fluidity of mouse synaptic plasma membranes. Biochim. Biophys. Acta 902, 385-393.

Smith, J.R., Laver, D.R. \& Coster, H.G.L. (1984) The conductance of lecithin bilayers: The dependence upon temperature. Chem. Phys. Lipids 34, 227-236.

Sunamoto, J., Iwamoto, K., Tezuka, T., Kadosaki, K. \& Kondo, H. (1983) Liposomal membranes. XIV. Fusion of liposomal membranes induced by polyisoprenoids as monitored by fluorescence quenching method. Chem. Phys. Lipids 33, 127-133.

Świeżewska, E., Sasak, W., Mańkowski, T., Jankowski, W., Vogtmann, T., Krajewska, I., Hertel, J., Skoczylas, E. \& Chojnacki, T. (1994) The search for plant polyprenols. Acta Biochim. Polon. 41, 221.

Tien, H.T. (1974) Bilayer Lipid Membranes (BLM): Theory and Practice. Marcel Dekker, New York.

Tomoaia-Cotişel, M., Zsako, J., Chifu, E. \& Quinn, P.J. (1987) Intermodular interactions in lipid/ carotenoid monolayers. Biochem. J. 248, 877882.

Valterson, C., van Duyn, G., Verkleij, A.J., Chojnacki, T., de Kruiff, B. \& Dallner, G. (1985) The influence of dolichol, dolichol esters and dolichyl phosphate on phospholipid polymorphism and fluidity in model membranes. J. Biol. Chem. 260, 2742-2751. 
Vigo, C., Grossman, S.H. \& Drost-Hansen, W. (1984) Interactions of dolichol and dolichyl phosphate with phospholipid bilayers. Biochim. Biophys. Acta 774, 221-226.

Wanke, M., Chojnacki, T. \& Świeżewska, E. (1998) The diversity of polyprenol pattern in leaves of fruit trees belonging to Rosaceae and Cornaceae. Acta Biochim. Polon. 45, 811-818.
Wood, W.G., Gorka, C., Williamson, L.S., Strong, R., Sun, G.Y. \& Schroeder, F. (1986) Dolichol alters dynamic and static properties of mouse synaptosomal plasma membranes. FEBS Lett. 205, 25-28.

Wood, W.G., Sun, G.Y. \& Schroeder, F. (1989) Membrane properties of dolichol in different age groups of mice. Chem. Phys. Lipids 51, 219-226. 